\section{Online fortbilden und CME-Punkte sammeln}

\section{Folgende Fortbildungen der MM W} stehen Ihnen derzeit auf

www.CME-Punkt.de zur Verfügung

(Auswahl):

\section{- Arzneimittelallergie/-intoleranz}

- FSME

- Asthma bronchiale

- Divertikulitis: Wann ist sie ein Fall für den Chirurgen?

- Alarmsymptom: Blut im Urin

- Narkosevorbereitung beim Hausarzt

- Stomaversorgung

Die Teilnahme ist für Sie völlig kostenfrei. Melden Sie sich heute noch an.

\section{UND SO GEHT'S:}

$\checkmark$ Auf www.CME-Punkt.degehen und sich einmal registrieren.

$\checkmark$ Dazu geben Sie persönliche Daten und Ihre Einheitliche Fortbildungsnummer (EFN) ein.

$\square$ Nach der Registrierung stehen Ihnen sämtliche CME-Fortbildungen der Zeitschriften von Urban\&Vogel kostenfrei zur Verfügung. Für Hausärzte und Internisten besonders interessant: MMW, Der Hausarzt, Medizinische Klinik, GastroNews, Cardiovasc, InFo Diabetologie, PneumoNews.

$\checkmark$ Wenn Sie eine Fortbildungseinheit bestehen (bis zu 3 Punkte), können Sie sich Ihre Teilnahmebestätigung direkt als PDF herunterladen.

$\checkmark$ Wir empfehlen, diese Bescheinigungen gesammelt bei Ihrer Landesärztekammer einzureichen.

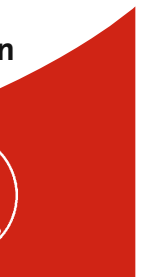

_ AKTUELLE MEDIZIN_LESERFORUM

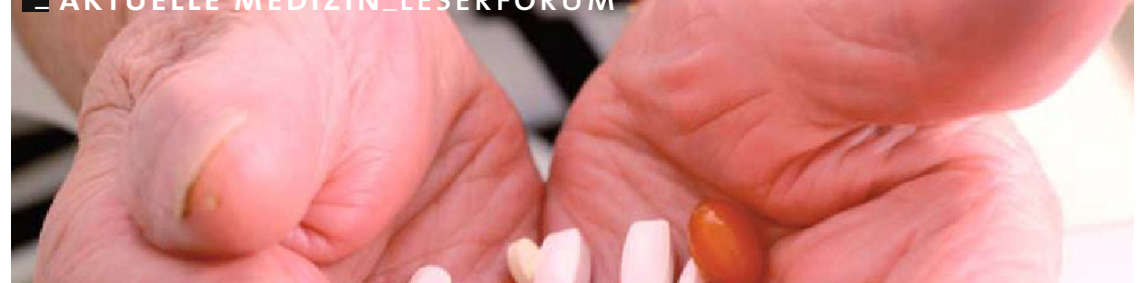

Therapieempfehlungen im Entlassungsbrief

\title{
Potenziell gefährlich
}

In unserem Schwerpunkt zur Polypharmakologie in MMW Nr. 10/2010, S. 31, demonstrierte unser Autor Dr. med. P. Stiefelhagen, Hachenburg, wie man Therapieempfehlungen, die ein Patient bei der Entlassung aus der Klinik mitbekommt, auf das Notwendigste reduziert. Dazu ein Leser:

— Die hier aufgeführte Therapieempfehlung bei Entlassung ist nicht nur ein Beispiel für Polypharmakotherapie, sondern auch potenziell gefährlich. Die gleichzeitige Gabe von PPI und Clopidogrel führt möglicherweise (bis zu 25\%) zu einer Wirkabschwächung des Clopidogrels, sodass die Häufigkeit von Stentthrombosen steigt. In einer kardiologisch-interventionell tätigen Abteilung sollte dies zum kleinen Einmaleins gehören.

- Dr. W. Kilchenstein per E-Mail

\section{Zusatzbeiträge der Krankenkassen}

\section{Ich fühle mich als Arzt und Patient verschaukelt}

\section{Zum Thema Zusatzbeiträge der} Krankenkassen erreichte uns folgender Leserbrief:

— Die BKK Dr. Oetker wirbt in einer sicher nicht ganz billigen Broschüre damit, dass sie 2010 keine Zusatzbeiträge erhebt. Wie man der gleichen Broschüre entnehmen kann, bietet diese BKK Leistungen an, die nur sehr entfernt etwas mit den Aufgaben einer Krankenkasse zu tun haben, z.B. Gesundheitsförderung in Sport- und Fitnessstudios, einen Online-Diät-Coach,
Mutter-(Vater-)Kind-Kuren usw. ... Trotzdem schafft es diese Kasse, ohne Zusatzbeiträge auszukommen. Da fühle ich mich sowohl als Arzt als auch als Patient von den anderen Kassen verschaukelt.

Es ist m.E. an der Zeit, die Machenschaften der Krankenkassen zu durchleuchten, um nicht ein ähnliches Desaster zu erleben wie bei den Banken.

\footnotetext{
- Dr. med. Udo Fuchs, Burgunderweg 11F, D-22453 Hamburg
} 